\title{
Outpatient Parathyroidectomy is Safe and Effective: A 7-year Review
}

\author{
Henry A Reinhart, $M D^{1^{*}}$, Samuel $K$ Snyder, $M D^{1}$, Michael D Bortz, $M D^{2}$, Susan V Stafford, $M D^{2}$, Victoria \\ E Wagner, $M D^{2}$, Camille W Graham, $M D^{2}$ and Xiaohui Wang, $P h D^{3}$
}

${ }^{1}$ Department of Surgery, UT Rio Grande Valley School of Medicine, Edinburg, TX, USA

${ }^{2}$ Department of Surgery, Baylor Scott and White Health, Temple, TX, USA

${ }^{3}$ School of Mathematical and Statistical Sciences, UT Rio Grande Valley, Edinburg, TX, USA

\begin{abstract}
Background: Minimally-invasive parathyroidectomy (MIP) techniques have made the procedure safer and more feasible as an outpatient parathyroidectomy (OP). We hypothesized that any type of parathyroidectomy is safe as an outpatient procedure and strive to characterize factors responsible for successful OP.
\end{abstract}

Methods: A retrospective analysis was performed of all parathyroidectomies between August 2009 and October 2016 with or without thyroidectomy to identify the type of parathyroid surgery, inpatient parathyroidectomy (IP) vs. OP status, postoperative complications, and resulting clinical outcomes.

Results: There were 672 parathyroidectomies, 51 IP (7.6\%) and 621 OP (92.4\%), consisting of 456 focused parathyroidectomies consisting of 1-2 gland resections (P) (67.9\%), 68 subtotal parathyroidectomies consisting of 3-3.5 gland resections (SP) (10.1\%), $125 \mathrm{P}$ with thyroidectomy (PT) (18.6\%), and $23 \mathrm{SP}$ with thyroidectomy (SPT) (3.4\%). Anesthesia included 369 (54.9\%) under general (GA) and 294 (43.8\%) under local anesthesia with monitored anesthesia care (L/ MAC). IP experienced more symptomatic hypocalcemia than OP $(13.7 \%$ vs. $4.3 \%)(p=0.01)$. Symptomatic hypocalcemia was more common after SP $(16.2 \%)$ or SPT $(17.4 \%)$ than P $(2.2 \%)$ or PT $(7.2 \%)(p<0.0001)$ and with GA $(8.1 \%)$ than L/ MAC (1.0\%) ( $p<0.0001)$. OP had a lower American Society of Anesthesia (ASA) score ( 2.5 mean) than IP (3.2 mean) ( $p<$ $0.0001)$. Three (5.9\%) recurrent laryngeal nerve $(R L N)$ injuries occurred with IP and six $(1.0 \%)$ with OP $(p=0.02)$ with only one permanent OP injury (0.16\%). RLN injury had no significant association with the extent of surgery ( $P, S P, P T$, and SPT), age, sex or type of anesthesia. One OP postoperative hematoma occurred $(0.16 \%)$ with a PT. There were no mortalities. Average OP postoperative time to discharge was 2.2 hours.

Conclusion: Outpatient parathyroid surgery is safe and should be applicable to nearly all patients, except those needing more extensive parathyroidectomy/thyroidectomy surgery or patients with greater comorbidities.

\section{Introduction}

Minimally-invasive parathyroidectomy (MIP) techniques have generally reduced the extent of parathyroidectomy surgery necessary to correct hyperparathyroidism. These techniques include a much smaller incision $(1-2 \mathrm{~cm})$ with exploration that is focused to the side of concern [1]. For the sake of this study MIP techniques did not include endoscopic methods. Neck ultrasound is the first line imaging modality for hyperparathyroidism but sestamibi imaging, CT of the neck including the $4 \mathrm{D}$ variant specific to parathyroid, MRI, and venous sampling can all aid in the localization of parathyroids [2]. Better image localization of abnormal parathyroid glands with preoperative ultrasound, parathyroid sestamibi scans, or 4D computerized tomography (CT) have allowed for a focused parathyroid gland exploration and less extensive surgery. This surgical approach can be facilitated with gamma probe localization after the injection of preoperative techne- tium sestamibi and/or intraoperative parathyroid hormone (PTH) monitoring. The focused surgical approach also facilitates performing parathyroidectomy under local anesthesia with monitored anesthesia care (L/MAC). MIP has made parathyroidectomy safer and more feasible as an outpatient parathyroidectomy (OP) procedure [3]. However, some patients still need a more extensive surgical exploration for the treat-

*Corresponding author: Henry Reinhart, MD, Department of Surgery, UT Rio Grande Valley School of Medicine, 2102 Treasure Hills Boulevard, Harlingen, TX 78550, USA, Tel: (956) 2961600

Accepted: February 20, 2021

Published online: February 22, 2021

Citation: Reinhart HA, Snyder SK, Bortz MD, et al. (2021) Outpatient Parathyroidectomy is Safe and Effective: A 7-year Review. J Surgical Endocrinol 3(1):67-72

Copyright: (C) 2021 Reinhart HA, et al. This is an open-access article distributed under the terms of the Creative Commons Attribution License, which permits unrestricted use, distribution, and reproduction in any medium, provided the original author and source are credited. 


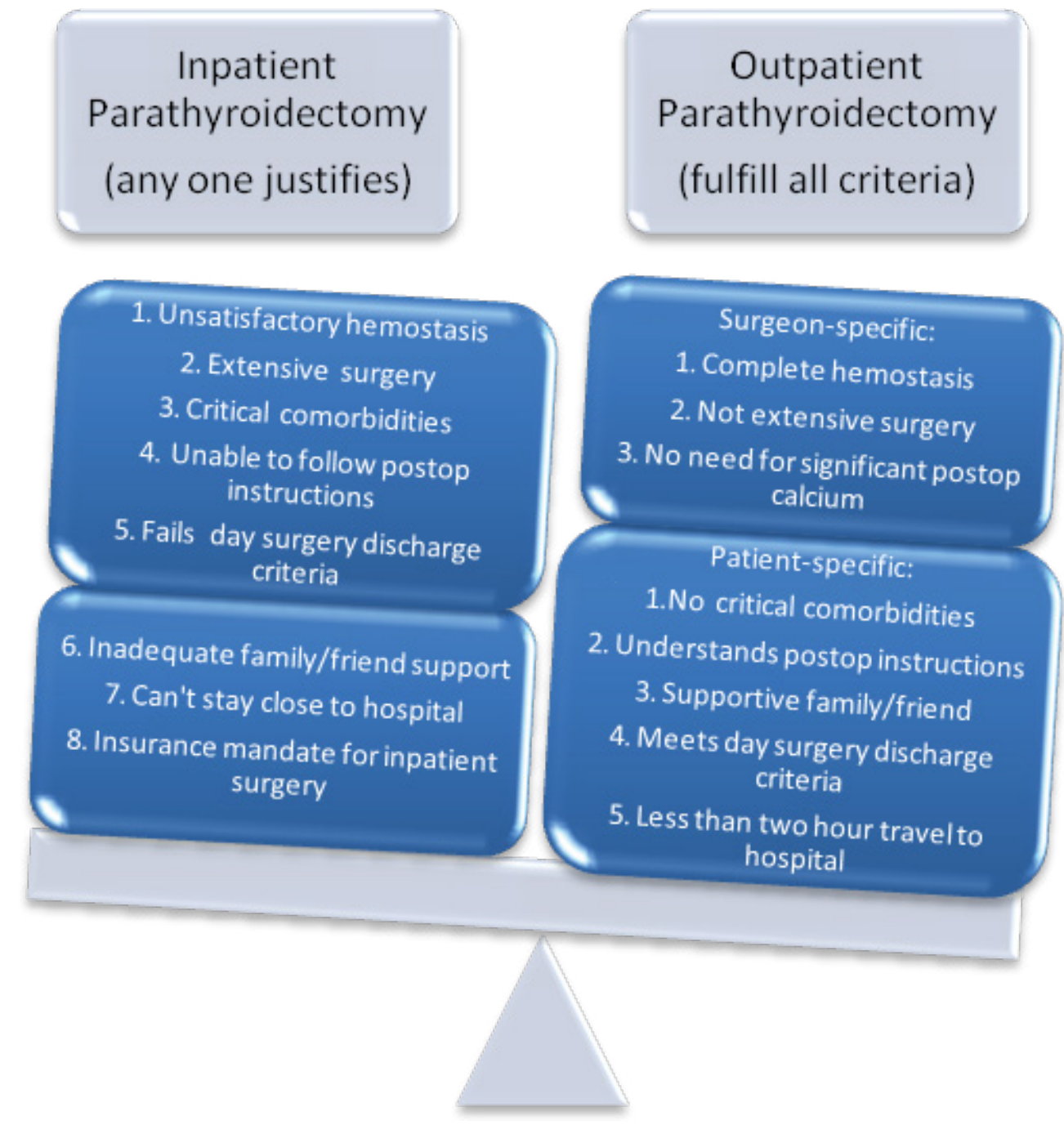

Figure 1: Factors influencing decision for inpatient vs. outpatient parathyroidectomy.

ment of multiglandular hyperparathyroidism and may need a combined thyroidectomy procedure. Outpatient thyroidectomy has been demonstrated to be safe and applicable to the majority of these procedures [4]. We hypothesized that any type of parathyroidectomy, with or without thyroidectomy, are safe as outpatient procedures. We strive to characterize those factors responsible for successful OP versus factors more likely to need inpatient parathyroidectomy (IP) management (Figure 1).

\section{Methods}

A seven-year retrospective analysis was performed at a high-volume center by a single surgeon after institutional review board approval from August 2009 through October 2016. All parathyroid procedures were included consisting of focused parathyroidectomies (P), subtotal parathyroidectomies (SP), P with thyroidectomy (PT), and SP with thyroidectomy (SPT). These were stratified as either IP or OP. Data collected included patient demographics, diagnosis, type of anesthesia, estimated blood loss (EBL), postoperative time to discharge, hypocalcemia with or without symptoms, recurrent laryngeal nerve (RLN) injury, hematoma formation, postoperative emergency room visits, and mortality. All parathyroidectomies for primary hyperparathyroidism (PHPT), secondary hyperparathyroidism (SHPT) and tertiary hyperparathyroidism (THPT) were included. P encompassed a 1-2 gland resection not limited to a single side and SP included a 3-3.5 gland resection. Thyroid surgery consisted of partial thyroidectomy (resection of less than one thyroid lobe), total lobectomy, or total thyroidectomy with or without central lymph node dissection. Data was collected from preoperative and postoperative documentation in the electronic record, operative reports, pathology records, and laboratory studies.

MIP was offered for PHPT, if the offending parathyroid gland could be visualized on ultrasound (US). If a parathyroid adenoma was not visualized on US, a parathyroid sestamibi scan along with a 4D CT scan was performed. If no gland was visualized on imaging, a unilateral 1-2 gland exploration or bilateral 4 gland exploration was undertaken still using MIP techniques. Preoperative injection of technetium sestamibi was given the morning of surgery for radio-guidance via gamma probe. Intraoperative parathyroid hormone (PTH) (third generation assay) was used to define the extent of surgery necessary to resolve the hyperparathyroidism. A pre-inci- 
sion PTH after anesthesia induction was drawn along with a pre-excision PTH (immediately prior to ligation of parathyroid vascular supply). A 5- and 10-minute post excision PTH was also drawn to assess for adequate resection. A decrease of $50 \%$ from the pre-incision level and return to normal was used for PHPT and a $90 \%$ decrease from the pre-incision level was used for SHPT or THPT for a 4 gland exploration. Diagnosis of hyperparathyroidism was biochemical with generally an elevated serum calcium level ( $10.4 \mathrm{mg} / \mathrm{dL}$ or higher) along with a high normal or elevated PTH (normal upper limit of 88 $\mathrm{pg} / \mathrm{mL})$.

Outpatient surgery was considered discharge on the same day as surgery from the postoperative recovery unit, while inpatient surgery included at least one night in the hospital. The postoperative day surgery recovery unit remained open until midnight, accepting patients from the Postoperative Anesthesia Care Unit (PACU) until 22:00 hours. A hematoma was clinically diagnosed as a significant postoperative neck swelling, with associated respiratory distress and/or dysphagia. These patients were transported urgently to the operating suite and decompressed in a head up position under local anesthesia. The hematoma was drained and any active bleeding controlled. Blood loss during surgery was estimated from used sponges as well as that collected in suction canisters. We visually identify the recurrent laryngeal nerve during surgery and perform a thyroid capsular dissection in order to prevent injuries. RLN injuries were identified intraoperatively during general anesthesia (GA) from the use of the RLN monitor utilizing an endotracheal tube with electrodes placed on the vocal cords. It was verified through the use of preoperative and postoperative laryngoscopy in all patients. Temporary nerve injuries were found to improve by six months while permanent injuries included a vocal cord palsy that lasted more than six months. Laryngoscopy before and after surgery is used for confirmation of injury.

Hypocalcemia was defined as a serum calcium level less than $8.5 \mathrm{mg} / \mathrm{dL}$. Symptomatic hypocalcemia represented related muscle cramping, and/or paresthesia in the distal extremities or lips. Our vitamin $D$ deficiency regimen included 50,000 international units of vitamin D2 (ergocalciferol) taken orally twice weekly in patients with Vitamin $D$ serum levels below 20 and once weekly for levels between 20-30. Once this was normalized, they were started on oral 2,000 international units of vitamin D3 daily. Patients with osteoporosis were given 1 tablet of $1250 \mathrm{mg}$ of calcium carbonate $(500 \mathrm{mg}$ of elemental calcium, Os-Cal) three times daily. PTH and calcium levels were followed starting in the PACU and continued in the outpatient setting. If patients were symptomatically hypocalcemic they were given 2 tabs of Os-Cal three times daily. If their PTH was below 5 they were also given $0.5 \mathrm{mcg}$ of calcitriol twice daily. Patients that underwent subtotal parathyroidectomy and experienced symptomatic hypocalcemia were treated with 2-4 g of calcium carbonate (Tums Ultra) as needed to relieve symptoms plus $0.5 \mathrm{mcg}$ of calcitriol twice daily.

Emergency room visits were recorded between discharge and postoperative day 30. Types of anesthesia included GA, local/MAC, or transition from local/MAC to GA due to patient intolerance. Postoperative time included the interval from leaving the operating room until discharge from the hospital or the day surgery recovery unit. Mortality was assessed up to 30 days postoperatively.

All patients were approached as potential candidates for outpatient surgery. Certain factors were evaluated prior to deciding on OP surgery. Surgeon specific factors for OP include satisfaction with the level of hemostasis after surgery, extent of surgery, and the need for significant postoperative calcium supplementation after surgery. Patient specific factors include associated comorbidities, patient's ability to follow directions, patient's support network, distance of their home from the hospital, and lastly insurance requirements. SP and SPT were considered for OP if they met the above requirements although each case required careful evaluation. Some patients were kept IP at their request and some insurances required admission for reimbursement purposes. There was no specific protocol used to decide on IP versus $\mathrm{OP}$ and each patient was carefully considered on a case-bycase basis. The decision for IP status was made after surgery occasionally after evaluating the surgeon specific factors. We did not collect the data for recurrent or persistent hyperparathyroidism.

Statistical analysis included descriptive statistics and pairwise analyses, including chi-square test or Fisher exact test, t-test and ANOVA. For continuous variables multiple linear regression analysis was utilized. Ordinal variables were analyzed via cumulative logit models. The EBL outcome required log-transformed linear regression along with pairwise analysis. American Society of Anesthesia (ASA) score used partial proportional odds model. Significance levels utilized were $0.05,0.01$, and 0.001 . Analyses were performed using $\mathrm{R}$ version 3.5.2 [5].

\section{Results}

The surgeries included 51 IP (7.6\%) and 621 OP (92.4\%) consisting of $456 \mathrm{P}(67.9 \%), 68 \mathrm{SP}(10.1 \%), 125$ PT $(18.6 \%)$, and 23 SPT (3.4\%). Gender was primarily female (504) (75\%) with only $25 \%$ being male (168) (25\%). The average age was 63. Anesthesia included 369 (54.9\%) utilizing general anesthesia (GA), 294 (43.8\%) utilizing local with monitored anesthesia care (L/MAC), and $9(1.3 \%)$ cases of L/MAC converted to GA due to patient intolerance. One OP postoperative hematoma occurred 10 hours after PT $(0.15 \%)$. OP was more likely to have a lower ASA score (2.5 mean) than IP (3.2 mean) $(p<0.0001)$. There were no mortalities. OP postoperative monitoring time averaged 2.2 hours.

IP experienced more symptomatic hypocalcemia (7) $(13.7 \%)$ than OP (27) $(4.3 \%)(p=0.01)$. GA (30) $(8.1 \%)$ was associated more with symptomatic hypocalcemia compared to L/MAC (3) (1.0\%) ( $p<0.0001)$. Patients who underwent $P$ (10) $(2.2 \%)$ were less likely to experience symptomatic hypocalcemia than those who had a SP (11) (16.2\%) ( $p=0.005)$. PT (9) (7.2\%) experienced less symptomatic hypocalcemia than SPT (4) $(17.4 \%)(p<0.0001)$. Patients who were younger were more likely to experience symptomatic hypocalcemia (51 years of age mean) when compared to patients that had no symptomatic hypocalcemia (64 years of age mean) ( $p=$ 
Citation: Reinhart HA, Snyder SK, Bortz MD, et al. (2021) Outpatient Parathyroidectomy is Safe and Effective: A 7-year Review. J Surgical Endocrinol 3(1):67-72

Table 1: Inpatients vs. outpatient characteristics.

\begin{tabular}{|l|l|l|l|}
\hline & IP & OP & Significance \\
\hline Total $(n, \%)$ & $51(7.6 \%)$ & $621(92.4 \%)$ & \\
\hline Symptomatic Hypocalcemia (n,\%) & $7(13.7 \%)$ & $27(4.3 \%)$ & $p=0.01$ \\
\hline Recurrent Laryngeal Nerve Injury (n, \%) & $3(5.9 \%)$ & $6(1 \%)$ & $p=0.025$ \\
\hline ASA Score (mean) & 3.2 & 2.5 & $p<0.0001$ \\
\hline Estimated Blood Loss (mean) & $54.1 \mathrm{~mL}$ & $13.8 \mathrm{~mL}$ & $\mathrm{p}=0.001$ \\
\hline ER Visits $(\mathrm{n}, \%)$ & $7(13.7 \%)$ & $50(8.1 \%)$ & $\mathrm{p}=0.185$ \\
\hline Hematoma $(\mathrm{n}, \%)$ & 0 & $1(0.2 \%)$ & $\mathrm{p}>0.05$ \\
\hline
\end{tabular}

ASA: American Society of Anesthesiologists; ER: Emergency Room

Table 2: Extent of surgery and associated findings.

\begin{tabular}{|l|l|l|l|l|l|}
\hline & P & SP & PT & SPT & Significance \\
\hline EBL (mean) & $7.1 \mathrm{~mL}$ & $19 \mathrm{~mL}$ & $41 \mathrm{~mL}$ & $72 \mathrm{~mL}$ & $\mathrm{p}<0.001$ \\
\hline ER Visits (n, \%) & $23,5 \%$ & $15,22 \%$ & $14,11 \%$ & $5,22 \%$ & $p<0.001$ \\
\hline Hypocaclcemia (n, \%) & $10,2 \%$ & $11,16 \%$ & $9,7 \%$ & $4,17 \%$ & $p<0.001$ \\
\hline RLN Injury (n, \%) & $8,2 \%$ & 0 & $2,2 \%$ & 0 & $p=0.5$ \\
\hline
\end{tabular}

P: Parathyroidectomy; SP: Subtotal Parathyroidectomy; PT: Parathyroidectomy with Thyroidectomy; SPT: Subtotal Parathyroidectomy with Thyroidectomy; EBL: Estimated Blood Loss; ER: Emergencu Room Visits; RLN: Recurrent Larygeal Nerve

Table 3: Extent of surgury and ASA score.

\begin{tabular}{|l|l|l|l|l|l|}
\hline & ASA 1 & ASA 2 & ASA 3 & ASA 4 & Total \\
\hline P & $8(1.8 \%)$ & $226(49.6 \%)$ & $207(45.4 \%)$ & $15(3.3 \%)$ & 456 \\
\hline SP & 0 & $9(13.2 \%)$ & $43(63.2 \%)$ & $16(23.5 \%)$ & 68 \\
\hline PT & $2(1.6 \%)$ & $62(49.6)$ & $58(46.4 \%)$ & $3(2.4 \%)$ & 125 \\
\hline SPT & 0 & $3(13 \%)$ & $10(43.5 \%)$ & $10(43.5 \%)$ & 23 \\
\hline
\end{tabular}

ASA: American Society of Anesthesiology; P: Parathyroidectomy; SP: Subtotal Parathyroidectomy; PT: Parathyroidectomy with Thyroidectomy; SPT: Subtotal Parathyroidectomy with Thyroidectom; No ASA 5 or 6 patients were included in the study

$0.001)$. There was no significant difference found when gen$\operatorname{der}(p=0.84)$ was evaluated with those that experienced symptomatic hypocalcemia.

When other factors were held constant, patients who had only a P (23) (5\%) were less likely to have postoperative ER visit than patients who underwent SP (15) $(22 \%)(p=0.001)$. In addition, surgeries where L/MAC was used (11) (3.7\%) were less likely to have a postoperative ER visit than those surgeries that utilized GA (45) (12.2\%) ( $p=0.001)$. There was no significant difference for IP vs. OP status $(p=0.12)$, gender $(p=0.94)$ and age $(p=0.1)$ when evaluating ER visits.

OP had fewer RLN injuries $(6,1 \%)$ than IP $(3,5.9 \%)(p=$ $0.025)$. There was one OP permanent recurrent laryngeal nerve injury $(0.16 \%)$. There was no association with the extent of surgery $(P, S P, P T, S P T)(p=0.5)$, age $(p=0.053)$, sex ( $p$ $=0.05)$ or type of anesthesia $(p=0.16)$ with RLN injury.

OP (mean $13.8 \mathrm{~mL}$ ) also had significantly less EBL when compared to IP (mean $54.1 \mathrm{~mL})(p=0.001)$. Patients who underwent $P$ (mean $7.1 \mathrm{~mL}$ ) lost less blood than SP (mean 19 $\mathrm{mL})$, PT (mean $41 \mathrm{~mL}$ ), and SPT (mean $72 \mathrm{~mL})(\mathrm{p}<0.001)$. As patient age increased EBL was found to decrease $(p=0.03)$. Lastly, patients that underwent L/MAC (mean $5.1 \mathrm{~mL}$ ) were likely to have a lower EBL than GA (mean $26 \mathrm{~mL})(\mathrm{p}<0.01)$ (Table 1, Table 2 and Table 3).

\section{Discussion}

We approach every parathyroidectomy as a possible OP candidate and the great majority of our surgeries were able to be performed as OP (92.4\%). Other studies have shown there are certain factors that should be considered prior to deciding on OP surgery [4]. Surgeon specific factors for OP include satisfaction with the level of hemostasis, extent of surgery, and the need for significant postoperative calcium supplementation. Patient specific factors include associated comorbidities, patient's ability to follow directions, patient's support network, distance of their home from the hospital, and lastly insurance requirements.

Hemostasis at the end of surgery is a critical factor for safe OP surgery. This can be difficult when there is bleeding near important structures such as the RLN and parathyroid but the importance of complete hemostasis cannot be overstated. All tools available, including titanium clips, suture ligation, and energy devices, need to be utilized to ensure that hemostasis is complete at the end of surgery. 
The extent of surgery should also be a surgeon specific consideration. If a patient requires SPT the surgeon should evaluate whether the benefits of OP surgery outweigh the risks such as symptomatic hypocalcemia or hematoma. Since hypocalcemia is a common cause of readmission after parathyroid surgery, the calcium supplementation needed after surgery should be addressed prior to deciding on OP surgery [6]. "Hungry bone syndrome" in patients with secondary and occasionally primary hyperparathyroidism can require a significant amount of calcium supplementation after surgery, sometimes making IP status a necessity [7]. In our institution, SHPT patients were able to receive dialysis the next day and were able to be seen daily at the endocrine surgery clinic. With the help of our outpatient center for IV medication infusion these patients were managed as OP with IV calcium gluconate if needed and sent home the same day. This practice may not translate to all institutions. We found that our IP experienced more symptomatic hypocalcemia than our OP. SP and SPT also experienced more symptomatic hypocalcemia when compared to $\mathrm{P}$ and $\mathrm{PT}$. In addition, patients undergoing GA were more likely to experience symptomatic hypocalcemia. All of these characteristics indicate that a more extensive surgery requiring GA and admission is more likely to experience symptomatic hypocalcemia.

Standardized postoperative calcium regimens help to decrease the likelihood of readmission, but do not eliminate the risk. Tailored regimens that supplement to measurable parameters such as bone density scores, preoperative calcium levels, and extent of surgery have been shown to diminish postoperative hypocalcemia [8]. These parameters remain an ongoing area of study. Our regimen included 50,000 international units of vitamin D2 (ergocalciferol) taken orally twice weekly in patients with serum levels below 20 and once weekly for levels between 20-30. Once this was normalized, they were started on oral 2,000 international units of vitamin D3 daily. Patients with osteoporosis were given 1 tablet of $1250 \mathrm{mg}$ of calcium carbonate (500 mg of elemental calcium, Os-Cal) three times daily. PTH and calcium levels were followed starting in the PACU and continued in the outpatient setting. Medication regimens were tailored to these findings.

Patients that require SP and SPT typically have more advanced disease such as renal dysfunction requiring dialysis, congestive heart failure, and other associated comorbidities. These patients may benefit from IP management in order to facilitate dialysis and medical management of such comorbidities. Unfortunately, we did not distinguish between the patients with secondary and tertiary hyperparathyroidism. In our cohort, IP had a higher ASA score. Thoughtful consideration needs to be given to the needs of the patient prior to deciding on OP surgery. Other patient specific considerations include the ability of patients to understand and follow directions. If the patient cannot perform specific tasks or does not have a support network available, they may benefit from IP monitoring. We recommend our patients stay within 2 hours of the hospital should any intervention be needed. Sometimes that requires an overnight hospital stay if the patient lives a significant distance from the hospital. Occasionally, an overnight admission was required for insurance reimburse- ment. In order to be discharged from the post-anesthesia care unit (PACU), patients need to meet the criteria based on the modified Aldrete Scoring system [9] along with having a flat, dry, cervical incision. Similar parameters have been set for outpatient thyroidectomy by the American Thyroid Association statement on outpatient thyroid surgery [10].

The incidence of postoperative hematoma after thyroid surgery has been shown to be $0.36-4.3 \%$ [11]. Our only hematoma occurred with PT in the OP setting. Although postoperative hematoma is rare after parathyroid surgery, the key to performing OP surgery safely lies in preventing this complication and safe management should it occur. This includes meticulous hemostasis during surgery and expeditious management should a hematoma develop postoperatively. Loupe magnification with headlight visualization helps to identify small areas of blood loss. The use of surgical clips, suture ligation, hemostatic agents and energy devices to control all areas of bleeding is essential to prevent hematoma, but despite the best effort, a hematoma can still occur postoperatively. For this reason, a system needs to be in place to manage this complication should it arise. Emergent bleeds that may obstruct the airway can occur shortly after surgery. These are likely to occur in the Post Anesthesia Care Unit (PACU) [12]. We observed our OP in the PACU for approximately 2.2 hours on average prior to hospital discharge. Some surgeons advocate for monitoring thyroidectomy patients for a minimum of 6 hours after surgery [13]. This would not have kept our single hematoma from being discharged. The hematoma was diagnosed at 10 hours post-surgery. The identification of a late hematoma requires patient and family education at the initial clinic visit and the day of surgery. The education should be concerning the signs and symptoms of a postoperative hematoma including a significant postoperative neck swelling with associated respiratory distress and/or dysphagia. Instructions need to be given on how to proceed if there is worry for hematoma [4]. In the past we utilized triage nurses to assess the patients concerns and escalate them to the surgeon if necessary. Currently, the widespread use of mobile phones allows patients to instantly transfer images and videos directly to the surgeon to help diagnose and triage. We currently give our mobile phone numbers to patients to notify us of any suspicion of a hematoma. We have utilized this system for several years without a problem. We also recommend that patients who need to travel long distances for surgery stay within a short distance from the hospital for the immediate postoperative period. Should a hematoma occur, the patient can usually be taken expeditiously to the operating suite while monitoring oxygen saturations. The incision can then be opened under local anesthesia in the semi-upright position, which is tolerated better than being supine. Once the hematoma is decompressed, the patient can be placed in the supine position and bleeding controlled. Intubation can be performed at his time if necessary. Previous studies have shown hematomas can be managed safely via this method [12].

Interestingly, we found older patients had decreased episodes of symptomatic hypocalcemia and the etiology is unclear. Perhaps, older patients are more likely to have a single 
parathyroid adenoma that requires less extensive surgery, which can then more likely be completed under L/MAC. One theory concludes that elderly patients are more likely to utilize calcium and/or vitamin D supplements preoperatively to prevent or treat osteoporosis which may prevent hypocalcemia [14]. Unfortunately, we did not make note of preoperative supplementation in our study.

ER visits were less likely in the $P$ group when compared to the SP group. The same was found when L/MAC was compared to GA. While there were many reasons for ER visits, the most common was symptomatic hypocalcemia. There was no significance seen between IP/OP status and ER visits. In summary, patients undergoing smaller and less complex surgeries tended to have fewer ER visits.

We found OP had fewer RLN injury than IP. A unilateral RLN injury is not an absolute indication for admission. Six of our injuries were OP. In order to accomplish this safely, we evaluate the respiratory and swallowing status prior to discharge. We also educate the patients on utilizing the chin tuck maneuver with fluids (swallowing via straw with the head tilted down and towards the site of injury). These patients can usually be discharged, if they are able to follow instructions and after a satisfactory period of evaluation to ensure no swallowing or breathing problems. Bilateral nerve injuries are a contraindication to OP parathyroid surgery.

We found OP surgery, focused P surgery, and surgery utilizing L/MAC all had less blood loss during surgery when compared to their counterparts. Increasing age was also found to be associated with less blood loss. One would expect that less invasive surgery would have less EBL, and the age discrepancy may represent more focused single parathyroid gland disease.

In summary, surgeon specific factors that should be considered for OP parathyroid surgery include satisfaction with the level of hemostasis, the extent of surgery and postoperative calcium needs. Patient specific factors include associated comorbidities, the patient's ability to follow directions, their support network, and the distance of home from the hospital. Lastly, some health coverage providers require inpatient monitoring with overnight observation.

The number of patient that underwent SP (68) and SPT (23) was much greater than the number of IP (51) although we did not stratify the number of IP that had a SP or SPT.

\section{Conclusion}

Outpatient parathyroid surgery is safe and should be applicable to nearly all patients.

\section{Acknowledgement}

There are no conflicts of interest. No funding was sought for this project.

\section{References}

1. Sosa JA, Udelsman R (2003) Minimally invasive parathyroidectomy. Surgical Oncology 12: 125-134.

2. Zafereo M, Yu J, Angelos P, et al. (2019) American Head and Neck Society Endocrine Surgery Section update on parathyroid imaging for surgical candidates with primary hyperparathyroidism. Head \& Neck 41: 2398-2409.

3. Noureldine SI, Gooi Z, Tufano RP (2015) Minimally invasive parathyroid surgery. Gland Surg 4: 410-419.

4. Reinhart HA, Snyder SK, Stafford SV, et al. (2018) Same day discharge after thyroidectomy is safe and effective. Surgery 164: 887-894.

5. R Core Team (2018) R: A language and environment for statistical computing. R Foundation for Statistical Computing, Vienna, Austria.

6. Roche AM, Brant JA, Chai RL (2018) Predictors of readmission and reoperation in patients undergoing parathyroidectomy for primary hyperparathyroidism. Otolaryngol Head Neck Surg 158: 828-834.

7. Jain N, Reilly RF (2017) Hungry bone syndrome. Curr Opin Nephrol Hypertens 26: 250-255.

8. Vasher M, Goodman A, Politz D, et al. (2010) Postoperative calcium requirements in 6,000 patients undergoing outpatient parathyroidectomy: Easily avoiding symptomatic hypocalcemia. J Am Coll Surg 211: 49-54.

9. Aldrete JA (1998) Modifications to the postanesthesia score for use in ambulatory surgery. J Perianesth Nurs 13: 148-155.

10. Terris DJ, Snyder S, Carneiro-Pla D, et al. (2013) American Thyroid Association statement on outpatient thyroidectomy. Thyroid 23: 1193-1202.

11. Pontin A, Pino A, Caruso E, et al. (2019) Postoperative Bleeding after Thyroid Surgery: Care Instructions. Sisli Etfal Hastan Tip Bul 53: 329-336.

12. Dixon JL, Snyder SK, Lairmore TC, et al. (2014) A novel method for the management of post-thyroidectomy or parathyroidectomy hematoma: A single-institution experience after over 4,000 central neck operations. World J Surg 38: 1262-1267.

13. Champault A, Vons C, Zilberman S, et al. (2009) How to perform a thyroidectomy in an outpatient setting. Langenbecks Arch Surg 394: 897-902.

14. Lindeman BM, Pesce CE, Tsai H-L, et al. (2014) Lower vitamin $D$ levels in surgical hyperparathyroidism versus thyroid patients. The American surgeon 80: 505-510.

DOI: $10.36959 / 608 / 450$

Copyright: (C) 2021 Reinhart HA, et al. This is an open-access article distributed under the terms of the Creative Commons Attribution License, which permits unrestricted use, distribution, and reproduction in any medium, provided the original author and source are credited. 